CRYSTALLOGRAPHIC COMMUNICATIONS

ISSN 2056-9890

Received 29 October 2018

Accepted 4 November 2018

Edited by A. J. Lough, University of Toronto, Canada

Keywords: crystal structure; chloranilic acid; ethyleneurea; imidazolidin-2-one; hydantoin; imidazolidine-2,4-dione; hydrogen bond.

CCDC references: 1876998; 1876997

Supporting information: this article has supporting information at journals.iucr.org/e

\section{Crystal structures of two hydrogen-bonded compounds of chloranilic acid-ethyleneurea (1/1) and chloranilic acid-hydantoin (1/2)}

\author{
Kazuma Gotoh and Hiroyuki Ishida*
}

Department of Chemistry, Faculty of Science, Okayama University, Okayama 700-8530, Japan. *Correspondence e-mail: ishidah@cc.okayama-u.ac.jp

The structures of the hydrogen-bonded 1:1 co-crystal of chloranilic acid (systematic name: 2,5-dichloro-3,6-dihydroxy-1,4-benzoquinone) with ethyleneurea (systematic name: imidazolidin-2-one), $\mathrm{C}_{6} \mathrm{H}_{2} \mathrm{Cl}_{2} \mathrm{O}_{4} \cdot \mathrm{C}_{3} \mathrm{H}_{6} \mathrm{~N}_{2} \mathrm{O}$, (I), and the 1:2 co-crystal of chloranilic acid with hydantoin (systematic name: imidazolidine-2,4-dione), $\mathrm{C}_{6} \mathrm{H}_{2} \mathrm{Cl}_{2} \mathrm{O}_{4} \cdot 2 \mathrm{C}_{3} \mathrm{H}_{4} \mathrm{~N}_{2} \mathrm{O}_{2}$, (II), have been determined at $180 \mathrm{~K}$. In the crystals of both compounds, the base molecules are in the lactam form and no acid-base interaction involving $\mathrm{H}$-atom transfer is observed. The asymmetric unit of (I) consists of two independent half-molecules of chloranilic acid, with each of the acid molecules lying about an inversion centre, and one ethyleneurea molecule. The asymmetric unit of (II) consists of one halfmolecule of chloranilic acid, which lies about an inversion centre, and one hydantoin molecule. In the crystal of (I), the acid and base molecules are linked via $\mathrm{O}-\mathrm{H} \cdots \mathrm{O}$ and $\mathrm{N}-\mathrm{H} \cdots \mathrm{O}$ hydrogen bonds, forming an undulating sheet structure parallel to the $a b$ plane. In (II), the base molecules form an inversion dimer via a pair of $\mathrm{N}-\mathrm{H}$. . O hydrogen bonds, and the base dimers are further linked through another $\mathrm{N}-\mathrm{H} \cdots \mathrm{O}$ hydrogen bond into a layer structure parallel to (101). The acid molecule and the base molecule are linked via an $\mathrm{O}-\mathrm{H} \cdots \mathrm{O}$ hydrogen bond.

\section{Chemical context}

Chloranilic acid, a dibasic acid with hydrogen-bond donor as well as acceptor groups, appears particularly attractive as a template for generating tightly bound self-assemblies with various organic bases, and also as a model compound for investigating hydrogen-transfer motions in $\mathrm{O}-\mathrm{H} \cdots \mathrm{N}$ and $\mathrm{N}-\mathrm{H} \cdots \mathrm{O}$ hydrogen-bonded systems (Zaman et al., 2004; Seliger et al., 2009; Asaji et al. 2010; Molčanov \& Kojić-Prodić, 2010). In the present study, we have prepared two hydrogenbonded compounds of chloranilic acid-ethyleneurea (1/1) and chloranilic acid-hydantoin (1/2) in order to extend our study on $D-\mathrm{H} \cdots A$ hydrogen bonding $(D=\mathrm{N}, \mathrm{O}$, or $\mathrm{C} ; A=\mathrm{N}$, O or $\mathrm{Cl}$ ) in chloranilic acid-organic base systems (Gotoh \& Ishida, $2017 a, b$, and references therein).<smiles>O=C1NCCN1</smiles>

OPEN ๑ ACCESS<smiles>O=C1C(O)=C(Cl)C(=O)C(O)=C1Cl</smiles>

(I)
2<smiles>O=C1CNC(=O)N1</smiles>

(II)<smiles>O=C1C(O)=C(Cl)C(=O)C(O)=C1Cl</smiles> 


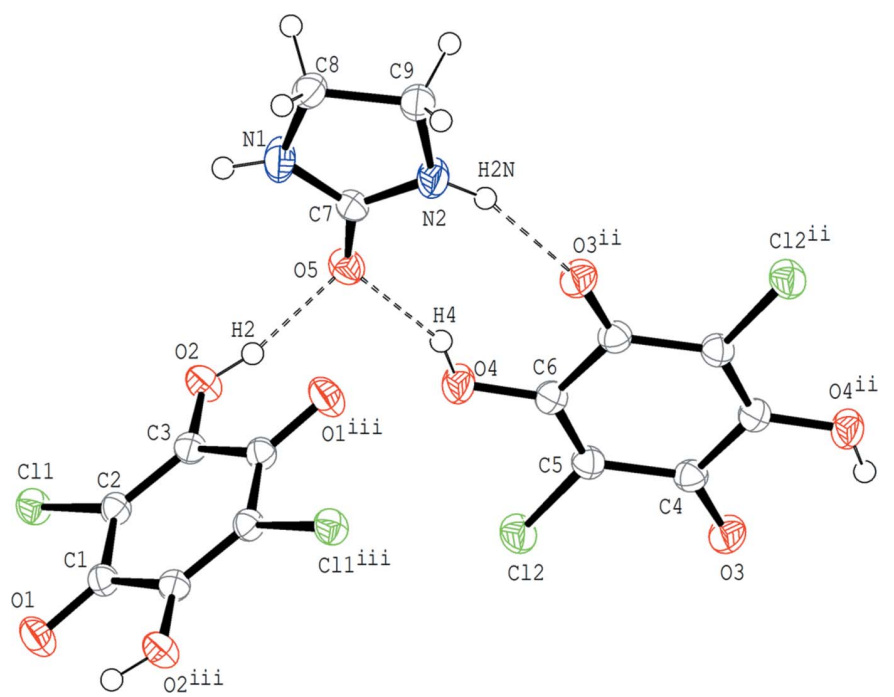

Figure 1

The molecular structure of compound (I), showing the atom-numbering scheme. Displacement ellipsoids of non-H atoms are drawn at the $50 \%$ probability level and $\mathrm{H}$ atoms are drawn as small spheres of arbitrary radii. $\mathrm{O}-\mathrm{H} \cdots \mathrm{O}$ and $\mathrm{N}-\mathrm{H} \cdots \mathrm{O}$ hydrogen bonds are shown by dashed lines. [Symmetry codes: (ii) $-x,-y+1,-z+1$; (iii) $-x+1,-y,-z+1$.]

\section{Structural commentary}

In compound (I), the base molecule is in the lactam form and no acid-base interaction involving $\mathrm{H}$-atom transfer is observed (Fig. 1). In the asymmetric unit, there is one ethyleneurea molecule and two crystallographically independent half-molecules of chloranilic acid, with each of the acid molecules lying about an inversion centre. The $\mathrm{O}$ atom of ethyleneurea participates in two $\mathrm{O}-\mathrm{H} \cdots \mathrm{O}$ hydrogen bonds as an acceptor for two $\mathrm{O}-\mathrm{H}$ groups of chloranilic acid $(\mathrm{O} 2-$ $\mathrm{H} 2 \cdots \mathrm{O} 5$ and $\mathrm{O} 4-\mathrm{H} 4 \cdots \mathrm{O} 5$; Table 1$)$. The base ring $(\mathrm{C} 7 / \mathrm{N} 1 /$ $\mathrm{C} 8 / \mathrm{C} 9 / \mathrm{N} 2)$ is essentially planar and makes dihedral angles of 88.75 (6) and $3.27(6)^{\circ}$, respectively, with the acid $\mathrm{C} 1-\mathrm{C} 3 / \mathrm{C} 1^{\mathrm{iii}}-$ $\mathrm{C}^{\mathrm{iii}}$ and $\mathrm{C} 4-\mathrm{C} 6 / \mathrm{C}^{\mathrm{ii}}-\mathrm{C}^{\mathrm{ii}}$ rings [symmetry codes: (ii) $-x$, $-y+1,-z+1 ;$ (iii) $-x+1,-y,-z+1]$.

In compound (II), the base molecule is also in the lactam form and no acid-base interaction involving $\mathrm{H}$-atom transfer is observed (Fig. 2). The chloranilic acid molecule is located on

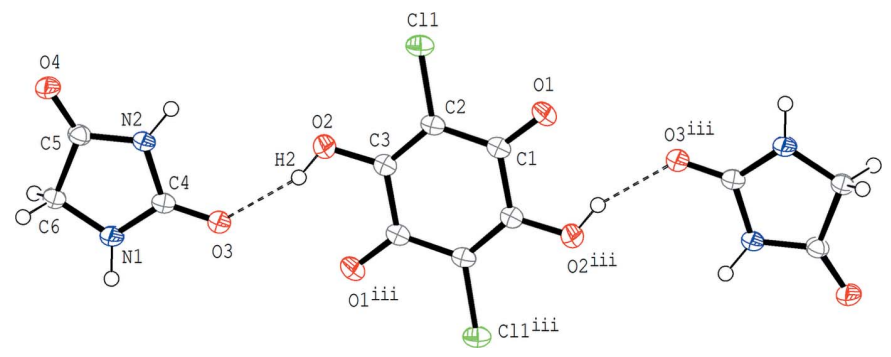

Figure 2

The molecular structure of compound (II), showing the atom-numbering scheme. Displacement ellipsoids of non-H atoms are drawn at the $50 \%$ probability level and $\mathrm{H}$ atoms are drawn as small spheres of arbitrary radii. $\mathrm{O}-\mathrm{H} \cdots \mathrm{O}$ hydrogen bonds are shown by dashed lines. [Symmetry code: (iii) $-x+\frac{1}{2},-y+\frac{5}{2},-z+1$.]
Table 1

Hydrogen-bond geometry $\left(\AA,^{\circ}\right)$ for (I).

\begin{tabular}{lllll}
\hline$D-\mathrm{H} \cdots A$ & $D-\mathrm{H}$ & $\mathrm{H} \cdots A$ & $D \cdots A$ & $D-\mathrm{H} \cdots A$ \\
\hline $\mathrm{O} 2-\mathrm{H} 2 \cdots \mathrm{O} 5$ & $0.81(2)$ & $1.82(2)$ & $2.6090(11)$ & $164(2)$ \\
$\mathrm{O} 4-\mathrm{H} 4 \cdots \mathrm{O} 5$ & $0.86(2)$ & $1.88(2)$ & $2.6635(12)$ & $151.0(19)$ \\
$\mathrm{N} 1-\mathrm{H} 1 N \cdots{ }^{\mathrm{i}}{ }^{\mathrm{i}}$ & $0.85(2)$ & $2.06(2)$ & $2.9003(15)$ & $168(2)$ \\
$\mathrm{N} 2-\mathrm{H} 2 N \cdots \mathrm{O}^{\mathrm{ii}}$ & $0.85(2)$ & $2.06(2)$ & $2.8654(15)$ & $158.6(17)$ \\
\hline
\end{tabular}

Symmetry codes: (i) $-x+2,-y,-z+1$; (ii) $-x,-y+1,-z+1$.

Table 2

Hydrogen-bond geometry ( $\left.\mathrm{A},{ }^{\circ}\right)$ for (II).

\begin{tabular}{lllll}
\hline$D-\mathrm{H} \cdots A$ & $D-\mathrm{H}$ & $\mathrm{H} \cdots A$ & $D \cdots A$ & $D-\mathrm{H} \cdots A$ \\
\hline $\mathrm{O} 2-\mathrm{H} 2 \cdots \mathrm{O} 3$ & $0.86(2)$ & $1.97(2)$ & $2.7917(15)$ & $160(2)$ \\
$\mathrm{N} 1-\mathrm{H} 1 N \cdots 3^{\mathrm{i}}$ & $0.91(2)$ & $2.00(2)$ & $2.8927(13)$ & $165(2)$ \\
$\mathrm{N} 2-\mathrm{H} 2 N \cdots 4^{\mathrm{ii}}$ & $0.91(2)$ & $1.85(2)$ & $2.7560(14)$ & $176(2)$ \\
\hline
\end{tabular}

Symmetry codes: (i) $-x+1,-y+1,-z+1$; (ii) $-x+\frac{1}{2}, y+\frac{1}{2},-z+\frac{1}{2}$

an inversion centre and the asymmetric unit consists of one hydantoin molecule and a half-molecule of chloranilic acid. The acid and base molecules are linked via an $\mathrm{O}-\mathrm{H} \cdots \mathrm{O}$ hydrogen bond $(\mathrm{O} 2-\mathrm{H} 2 \cdots \mathrm{O} 3$; Table 2$)$, forming a centrosymmetric $1: 2$ aggregate of the acid and the base. The 1:2 unit is approximately planar with a dihedral angle of $5.42(5)^{\circ}$ between the acid and base rings.

\section{Supramolecular features}

In the crystal of compound (I), the acid and base molecules are alternately arranged through $\mathrm{O}-\mathrm{H} \cdots \mathrm{O}$ and $\mathrm{N}-\mathrm{H} \cdots \mathrm{O}$ hydrogen bonds $\left(\mathrm{O} 4-\mathrm{H} 4 \cdots \mathrm{O} 5, \mathrm{~N} 1-\mathrm{H} 1 N \cdots \mathrm{O} 1^{\mathrm{i}}, \mathrm{N} 2-\right.$ $\mathrm{H} 2 \mathrm{H} \cdots \mathrm{O} 3^{\mathrm{ii}}$; symmetry codes as in Table 1 ), forming an undulating tape structure along [3ī0]. The tapes are stacked along the $a$ axis via another $\mathrm{O}-\mathrm{H} \cdots \mathrm{O}$ hydrogen bond $(\mathrm{O} 2-$ $\mathrm{H} 2-\mathrm{O} 5$; Table 1) into a sheet structure parallel to the $a b$ plane (Fig. 3).

In the crystal of (II), two adjacent base molecules, which are related by an inversion centre, form a dimer via a pair of $\mathrm{N}-$ $\mathrm{H} \cdots \mathrm{O}$ hydrogen bonds $\left(\mathrm{N} 1-\mathrm{H} 1 N \cdots \mathrm{O} 3^{\mathrm{i}}\right.$; symmetry code as

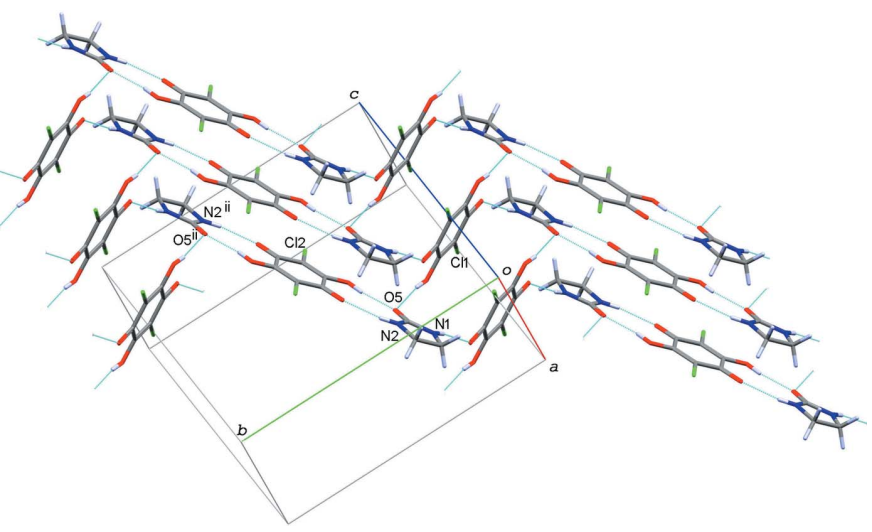

Figure 3

A partial packing diagram of compound (I), showing the undulating sheet structure formed by $\mathrm{O}-\mathrm{H} \cdots \mathrm{O}$ and $\mathrm{N}-\mathrm{H} \cdots \mathrm{O}$ hydrogen bonds (lightblue dotted lines). [Symmetry code: (ii) $-x,-y+1,-z+1$.] 


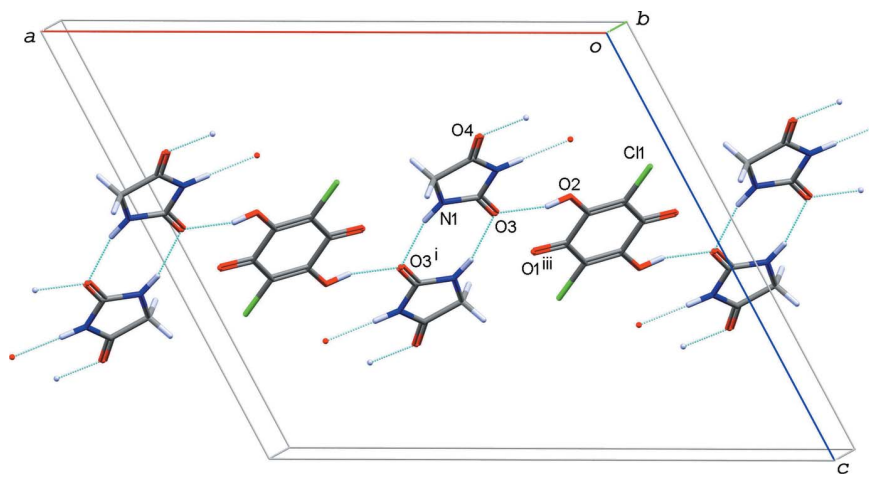

Figure 4

A partial packing diagram of compound (II) viewed approximately along the $b$ axis, showing a hydrogen-bonded tape structure formed by acid molecules and pairs of base molecules. $\mathrm{O}-\mathrm{H} \cdots \mathrm{O}$ and $\mathrm{N}-\mathrm{H} \cdots \mathrm{O}$ hydrogen bonds are shown by light-blue dotted lines. [Symmetry codes: (i) $-x+1,-y+1,-z+1$; (iii) $-x+\frac{1}{2},-y+\frac{5}{2},-z+1$.]

in Table 2), and the base dimer and the acid molecule are alternately linked through an $\mathrm{O}-\mathrm{H} \cdots \mathrm{O}$ hydrogen bond $(\mathrm{O} 2-\mathrm{H} 2 \cdots \mathrm{O} 3$; Table 2$)$, forming a flat tape structure along the $a$-axis direction (Fig. 4). The base dimers are assembled via another $\mathrm{N}-\mathrm{H} \cdots \mathrm{O}$ hydrogen bond $\left(\mathrm{N} 2-\mathrm{H} 2 \mathrm{~N} \cdots \mathrm{O} 4^{\mathrm{ii}}\right.$; symmetry code as in Table 2), forming a layer parallel to (101) as shown in Fig. 5. The $\mathrm{O}-\mathrm{H} \cdots \mathrm{O}$ hydrogen bond $(\mathrm{O} 2-$ $\mathrm{H} 2$...O3; Table 2) formed between the acid and base molecules links the layers.

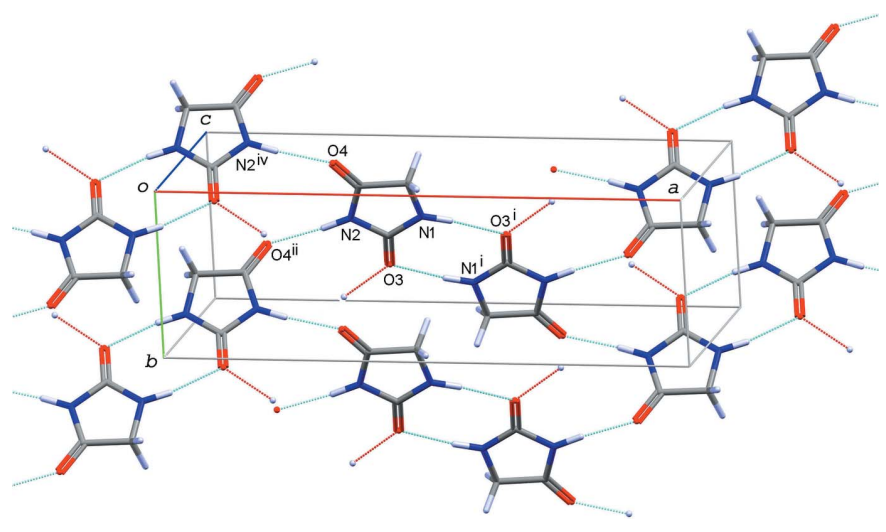

Figure 5

A partial packing diagram of compound (II), showing hydrogen-bonding scheme in the layer formed by base molecules. $\mathrm{N}-\mathrm{H} \cdots \mathrm{O}$ hydrogen bonds between the base molecules are shown by light-blue dotted lines, while $\mathrm{O}-\mathrm{H} \cdots \mathrm{O}$ hydrogen bonds between the base and acid molecules are shown by red dotted lines. [Symmetry codes: (i) $-x+1,-y+1,-z+1$; (ii) $-x+\frac{1}{2}, y+\frac{1}{2},-z+\frac{1}{2}$; (iv) $-x+\frac{1}{2}, y-\frac{1}{2},-z+\frac{1}{2}$.]

\section{Database survey}

A search of the Cambridge Structural Database (Version 5.39, last update August 2018; Groom et al., 2016) for organic crystals of chloranilic acid with lactam-form base molecules gave ten hits. In the seven crystals of these compounds, $\mathrm{O}-$ $\mathrm{H}$... O hydrogen bonds between the $\mathrm{O}-\mathrm{H}$ group of chloranilic acid and the carbonyl group of base are observed

Table 3

Experimental details.

Crystal data

Chemical formula

$M_{\mathrm{r}}$

Crystal system, space group

Temperature (K)

$a, b, c(\AA)$

$\beta\left({ }^{\circ}\right)$

$V\left(\AA^{3}\right)$

$Z$

Radiation type

$\mu\left(\mathrm{mm}^{-1}\right)$

Crystal size (mm)

Data collection

Diffractometer

Absorption correction

$T_{\min }, T_{\max }$

No. of measured, independent and observed $[I>2 \sigma(I)]$ reflections

$R_{\text {int }}$

$(\sin \theta / \lambda)_{\max }\left(\AA^{-1}\right)$

$\mathrm{C}_{6} \mathrm{H}_{2} \mathrm{Cl}_{2} \mathrm{O}_{4} \cdot \mathrm{C}_{3} \mathrm{H}_{6} \mathrm{~N}_{2} \mathrm{O}$
295.08
Monoclinic, $P P_{1} / c$
180
$5.0180(4), 14.6142(10), 15.8882(11)$
$105.563(3)$
$1122.43(15)$
4
Mo $K \alpha$
0.59
$0.45 \times 0.29 \times 0.23$

Rigaku R-AXIS RAPIDII

Numerical (NUMABS; Higashi, 1999)

$0.716,0.873$

$21546,3266,3040$

0.057

0.704

\author{
$\mathrm{C}_{6} \mathrm{H}_{2} \mathrm{Cl}_{2} \mathrm{O}_{4} \cdot 2 \mathrm{C}_{3} \mathrm{H}_{4} \mathrm{~N}_{2} \mathrm{O}_{2}$ \\ 409.14 \\ Monoclinic, $C 2 / c$ \\ 180 \\ 19.5690 (8), 5.18661 (10), 16.6103 (3) \\ $117.965(3)$ \\ $1489.03(8)$ \\ 4 \\ Mo $K \alpha$ \\ 0.49 \\ $0.49 \times 0.33 \times 0.24$
}

\section{Rigaku R-AXIS RAPIDII}

Numerical (NUMABS; Higashi, 1999)

$0.808,0.888$

14622, 2181, 2029

0.072

0.704

Refinement

$R\left[F^{2}>2 \sigma\left(F^{2}\right)\right], w R\left(F^{2}\right), S$

No. of reflections

No. of parameters

$\mathrm{H}$-atom treatment

$\Delta \rho_{\max }, \Delta \rho_{\min }\left(\mathrm{e} \AA^{-3}\right)$

\begin{tabular}{ll}
$0.033,0.092,1.07$ & $0.036,0.100,1.08$ \\
3266 & 2181 \\
179 & 130 \\
$\begin{array}{l}\mathrm{H} \text { atoms treated by a mixture of independent } \\
\text { and constrained refinement }\end{array}$ & $\mathrm{H}$ atoms treated by a mixture of independent \\
$0.54,-0.31$ & and constrained refinement \\
\hline
\end{tabular}

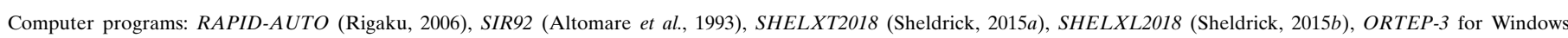
(Farrugia, 2012), Mercury (Macrae et al., 2006), CrystalStructure (Rigaku, 2018) and PLATON (Spek, 2015). 
[refcodes ACOJIO (Gotoh \& Ishida, 2017a), AJAGIB (Luo \& Palmore, 2002), HUFZUE (Jasinski et al., 2010), ODIHIU, SADTIC, SADTOI and SADTUO (Gotoh \& Ishida, 2011)]. In particular, the compounds of chloranilic acid with 2-pyridone (ACOJIO), gabapentin-lactum (HUFZUE), pyrrolidin2-one (ODIHIU) and piperidin-2-one (SADTUO) show short $\mathrm{O}-\mathrm{H} \cdots \mathrm{O}$ hydrogen bonds $(\mathrm{O} \cdots \mathrm{O}$ shorter than $2.5 \AA)$. In the $\mathrm{O}-\mathrm{H} \cdots \mathrm{O}$ hydrogen bond $[\mathrm{O} \cdots \mathrm{O}=2.4484(10) \AA]$ of chloranilic acid-piperidin-2-one (1/2) (SADTUO), the $\mathrm{H}$ atom is disordered over two positions.

\section{Synthesis and crystallization}

Single crystals of compound (I) were obtained by slow evaporation from an acetonitrile solution $(150 \mathrm{ml})$ of chloranilic acid $(330 \mathrm{mg})$ with ethyleneurea $(140 \mathrm{mg})$ at room temperature. Crystals of compound (II) were obtained by slow evaporation from an acetonitrile solution $(250 \mathrm{ml})$ of chloranilic acid (350 $\mathrm{mg}$ ) with hydantoin (340 mg) at room temperature.

\section{Refinement}

Crystal data, data collection and structure refinement details are summarized in Table 3. All $\mathrm{H}$ atoms in compounds (I) and (II) were found in difference Fourier maps. The O- and $\mathrm{N}$ bound $\mathrm{H}$ atoms were freely refined. $\mathrm{C}$-bound $\mathrm{H}$ atoms were positioned geometrically $(\mathrm{C}-\mathrm{H}=0.99 \AA)$ and were treated as riding with $U_{\text {iso }}(\mathrm{H})=1.2 U_{\text {eq }}(\mathrm{C})$.

\section{References}

Altomare, A., Cascarano, G., Giacovazzo, C. \& Guagliardi, A. (1993). J. Appl. Cryst. 26, 343-350.

Asaji, T., Seliger, J., Žagar, V. \& Ishida, H. (2010). Magn. Reson. Chem. 48, 531-536.

Farrugia, L. J. (2012). J. Appl. Cryst. 45, 849-854.

Gotoh, K. \& Ishida, H. (2011). Acta Cryst. C67, o500-o504.

Gotoh, K. \& Ishida, H. (2017a). Acta Cryst. E73, 1546-1550.

Gotoh, K. \& Ishida, H. (2017b). Acta Cryst. E73, 1840-1844.

Groom, C. R., Bruno, I. J., Lightfoot, M. P. \& Ward, S. C. (2016). Acta Cryst. B72, 171-179.

Higashi, T. (1999). NUMABS. Rigaku Corporation, Tokyo, Japan.

Jasinski, J. P., Butcher, R. J., Hakim Al-arique, Q. N. M., Yathirajan, H. S. \& Narayana, B. (2010). Acta Cryst. E66, o163-o164.

Luo, T. M. \& Palmore, G. T. R. (2002). Cryst. Growth Des. 2, 337-350.

Macrae, C. F., Edgington, P. R., McCabe, P., Pidcock, E., Shields, G. P., Taylor, R., Towler, M. \& van de Streek, J. (2006). J. Appl. Cryst. 39, 453-457.

Molčanov, K. \& Kojić-Prodić, B. (2010). CrystEngComm, 12, 925-939. Rigaku (2006). RAPID-AUTO. Rigaku Corporation, Tokyo, Japan. Rigaku (2018). CrystalStructure. Rigaku Corporation, Tokyo, Japan. Seliger, J., Žagar, V., Gotoh, K., Ishida, H., Konnai, A., Amino, D. \& Asaji, T. (2009). Phys. Chem. Chem. Phys. 11, 2281-2286.

Sheldrick, G. M. (2015a). Acta Cryst. A71, 3-8.

Sheldrick, G. M. (2015b). Acta Cryst. C71, 3-8.

Spek, A. L. (2015). Acta Cryst. C71, 9-18.

Zaman, Md. B., Udachin, K. A. \& Ripmeester, J. A. (2004). Cryst. Growth Des. 4, 585-589. 


\section{supporting information}

Acta Cryst. (2018). E74, 1727-1730 [https://doi.org/10.1107/S205698901801561X]

\section{Crystal structures of two hydrogen-bonded compounds of chloranilic acid- ethyleneurea (1/1) and chloranilic acid-hydantoin (1/2)}

\section{Kazuma Gotoh and Hiroyuki Ishida}

\section{Computing details}

For both structures, data collection: RAPID-AUTO (Rigaku, 2006); cell refinement: RAPID-AUTO (Rigaku, 2006); data reduction: RAPID-AUTO (Rigaku, 2006). Program(s) used to solve structure: SIR92 (Altomare et al., 1993) for (I); SHELXT2018 (Sheldrick, 2015a) for (II). For both structures, program(s) used to refine structure: SHELXL2018 (Sheldrick, 2015b); molecular graphics: ORTEP-3 for Windows (Farrugia, 2012) and Mercury (Macrae et al., 2006); software used to prepare material for publication: CrystalStructure (Rigaku, 2018) and PLATON (Spek, 2015).

2,5-Dichloro-3,6-dihydroxy-1,4-benzoquinone-imidazolidin-2-one (1/1) (I)

Crystal data

$$
\begin{aligned}
& \mathrm{C}_{6} \mathrm{H}_{2} \mathrm{Cl}_{2} \mathrm{O}_{4} \cdot \mathrm{C}_{3} \mathrm{H}_{6} \mathrm{~N}_{2} \mathrm{O} \\
& M_{r}=295.08 \\
& \text { Monoclinic, } P 2_{1} / c \\
& a=5.0180(4) \AA \\
& b=14.6142(10) \AA \\
& c=15.8882(11) \AA \\
& \beta=105.563(3)^{\circ} \\
& V=1122.43(15) \AA^{3} \\
& Z=4
\end{aligned}
$$

Data collection

Rigaku R-AXIS RAPIDII diffractometer

Detector resolution: 10.000 pixels $\mathrm{mm}^{-1}$

$\omega$ scans

Absorption correction: numerical

(NUMABS; Higashi, 1999)

$T_{\min }=0.716, T_{\max }=0.873$

21546 measured reflections

\section{Refinement}

Refinement on $F^{2}$

$R\left[F^{2}>2 \sigma\left(F^{2}\right)\right]=0.033$

$w R\left(F^{2}\right)=0.092$

$S=1.07$

3266 reflections

179 parameters

0 restraints

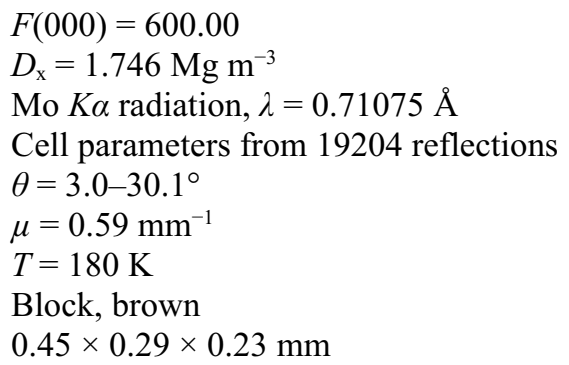

3266 independent reflections 3040 reflections with $I>2 \sigma(I)$

$R_{\text {int }}=0.057$

$\theta_{\max }=30.0^{\circ}, \theta_{\min }=3.0^{\circ}$

$h=-7 \rightarrow 7$

$k=-20 \rightarrow 19$

$l=-21 \rightarrow 22$

Primary atom site location: structure-invariant direct methods

Secondary atom site location: difference Fourier map

Hydrogen site location: mixed

$\mathrm{H}$ atoms treated by a mixture of independent and constrained refinement 
$w=1 /\left[\sigma^{2}\left(F_{\mathrm{o}}^{2}\right)+(0.0573 P)^{2}+0.2007 P\right]$

where $P=\left(F_{\mathrm{o}}^{2}+2 F_{\mathrm{c}}^{2}\right) / 3$

$(\Delta / \sigma)_{\max }=0.001$

$$
\Delta \rho_{\max }=0.54 \text { e } \AA^{-3}
$$

Special details

Geometry. All esds (except the esd in the dihedral angle between two 1.s. planes) are estimated using the full covariance matrix. The cell esds are taken into account individually in the estimation of esds in distances, angles and torsion angles; correlations between esds in cell parameters are only used when they are defined by crystal symmetry. An approximate (isotropic) treatment of cell esds is used for estimating esds involving 1.s. planes.

Fractional atomic coordinates and isotropic or equivalent isotropic displacement parameters $\left(A^{2}\right)$

\begin{tabular}{lllll}
\hline & $x$ & $y$ & $z$ & $U_{\text {iso }} * / U_{\text {eq }}$ \\
\hline C11 & $1.06653(5)$ & $0.00389(2)$ & $0.64704(2)$ & $0.02517(9)$ \\
C12 & $0.28647(6)$ & $0.43040(2)$ & $0.69108(2)$ & $0.03101(9)$ \\
O1 & $0.64834(18)$ & $-0.14266(6)$ & $0.60507(6)$ & $0.03198(19)$ \\
O2 & $0.83758(17)$ & $0.15225(5)$ & $0.51887(5)$ & $0.02612(17)$ \\
O3 & $-0.17821(19)$ & $0.55990(6)$ & $0.63245(6)$ & $0.03138(19)$ \\
O4 & $0.43441(18)$ & $0.37722(6)$ & $0.52805(6)$ & $0.03039(19)$ \\
O5 & $0.70481(17)$ & $0.29928(5)$ & $0.42370(5)$ & $0.02644(17)$ \\
N1 & $0.8521(2)$ & $0.24152(9)$ & $0.30842(7)$ & $0.0372(3)$ \\
N2 & $0.4933(2)$ & $0.33134(8)$ & $0.27881(7)$ & $0.0323(2)$ \\
C1 & $0.5873(2)$ & $-0.07583(7)$ & $0.55745(7)$ & $0.02118(19)$ \\
C2 & $0.7619(2)$ & $0.00483(7)$ & $0.56565(7)$ & $0.02058(19)$ \\
C3 & $0.6846(2)$ & $0.07805(7)$ & $0.51226(6)$ & $0.02064(19)$ \\
C4 & $-0.0902(2)$ & $0.53136(7)$ & $0.57314(7)$ & $0.0233(2)$ \\
C5 & $0.1390(2)$ & $0.46653(7)$ & $0.58624(7)$ & $0.0238(2)$ \\
C6 & $0.2263(2)$ & $0.43578(7)$ & $0.51801(7)$ & $0.0236(2)$ \\
C7 & $0.6858(2)$ & $0.29121(7)$ & $0.34329(7)$ & $0.0224(2)$ \\
C8 & $0.7865(2)$ & $0.24974(8)$ & $0.21442(8)$ & $0.0275(2)$ \\
H8A & 0.737465 & 0.189643 & 0.185683 & $0.033^{*}$ \\
H8B & 0.943169 & 0.276222 & 0.195803 & $0.033^{*}$ \\
C9 & $0.5369(3)$ & $0.31466(9)$ & $0.19376(8)$ & $0.0325(2)$ \\
H9A & 0.578932 & 0.372186 & 0.166986 & $0.039^{*}$ \\
H9B & 0.372898 & 0.285493 & 0.153938 & $0.039^{*}$ \\
H1N & $0.989(4)$ & $0.2121(14)$ & $0.3405(14)$ & $0.059(6)^{*}$ \\
H2N & $0.381(4)$ & $0.3687(13)$ & $0.2915(12)$ & $0.041(5)^{*}$ \\
H2 & $0.766(4)$ & $0.1933(16)$ & $0.4861(14)$ & $0.056(6)^{*}$ \\
H4 & $0.476(4)$ & $0.3613(13)$ & $0.4811(13)$ & $0.045(5)^{*}$ \\
& & & & \\
& & & &
\end{tabular}

Atomic displacement parameters $\left(\AA^{2}\right)$

\begin{tabular}{lllllll}
\hline & $U^{11}$ & $U^{22}$ & $U^{33}$ & $U^{12}$ & $U^{13}$ & $U^{23}$ \\
\hline C11 & $0.02251(14)$ & $0.02897(15)$ & $0.02081(14)$ & $-0.00049(8)$ & $0.00027(10)$ & $0.00183(8)$ \\
C12 & $0.03685(16)$ & $0.03457(16)$ & $0.02165(15)$ & $0.00812(10)$ & $0.00789(11)$ & $0.00275(9)$ \\
O1 & $0.0284(4)$ & $0.0277(4)$ & $0.0345(4)$ & $-0.0009(3)$ & $-0.0007(3)$ & $0.0114(3)$ \\
O2 & $0.0273(4)$ & $0.0226(4)$ & $0.0252(4)$ & $-0.0031(3)$ & $0.0014(3)$ & $0.0039(3)$ \\
O3 & $0.0355(4)$ & $0.0356(4)$ & $0.0262(4)$ & $0.0082(3)$ & $0.0138(3)$ & $-0.0009(3)$ \\
O4 & $0.0333(4)$ & $0.0350(4)$ & $0.0243(4)$ & $0.0124(3)$ & $0.0100(3)$ & $0.0016(3)$
\end{tabular}


supporting information

$\begin{array}{lllllll}\text { O5 } & 0.0319(4) & 0.0244(4) & 0.0230(4) & 0.0017(3) & 0.0071(3) & 0.0006(3) \\ \mathrm{N} 1 & 0.0378(6) & 0.0468(6) & 0.0272(5) & 0.0224(5) & 0.0093(4) & 0.0057(4) \\ \mathrm{N} 2 & 0.0336(5) & 0.0411(5) & 0.0240(5) & 0.0163(4) & 0.0106(4) & 0.0045(4) \\ \mathrm{C} 1 & 0.0207(4) & 0.0220(4) & 0.0204(4) & 0.0018(3) & 0.0047(3) & 0.0012(3) \\ \mathrm{C} 2 & 0.0192(4) & 0.0236(5) & 0.0178(4) & 0.0012(3) & 0.0029(3) & 0.0002(3) \\ \mathrm{C} 3 & 0.0215(4) & 0.0221(4) & 0.0181(4) & 0.0003(3) & 0.0049(3) & -0.0006(3) \\ \mathrm{C} 4 & 0.0253(5) & 0.0233(5) & 0.0225(5) & -0.0001(4) & 0.0083(4) & -0.0004(4) \\ \mathrm{C} 5 & 0.0260(5) & 0.0245(5) & 0.0213(5) & 0.0019(4) & 0.0070(4) & 0.0010(4) \\ \text { C6 } & 0.0244(5) & 0.0231(5) & 0.0240(5) & 0.0016(3) & 0.0077(4) & 0.0002(3) \\ \text { C7 } & 0.0239(4) & 0.0191(4) & 0.0246(5) & -0.0005(3) & 0.0070(4) & 0.0021(3) \\ \text { C8 } & 0.0254(5) & 0.0315(5) & 0.0275(5) & 0.0034(4) & 0.0100(4) & -0.0007(4) \\ \text { C9 } & 0.0346(6) & 0.0416(6) & 0.0240(5) & 0.0125(5) & 0.0127(4) & 0.0075(4)\end{array}$

Geometric parameters $\left(\AA,{ }^{\circ}\right)$

\begin{tabular}{|c|c|c|c|}
\hline $\mathrm{C} 11-\mathrm{C} 2$ & $1.7169(10)$ & $\mathrm{N} 2-\mathrm{C} 9$ & $1.4464(15)$ \\
\hline $\mathrm{Cl} 2-\mathrm{C} 5$ & $1.7145(11)$ & $\mathrm{N} 2-\mathrm{H} 2 \mathrm{~N}$ & 0.849 (19) \\
\hline $\mathrm{O} 1-\mathrm{C} 1$ & $1.2230(13)$ & $\mathrm{C} 1-\mathrm{C} 2$ & $1.4537(14)$ \\
\hline $\mathrm{O} 2-\mathrm{C} 3$ & $1.3165(12)$ & $\mathrm{C} 1-\mathrm{C} 3^{\mathrm{i}}$ & $1.5092(14)$ \\
\hline $\mathrm{O} 2-\mathrm{H} 2$ & $0.81(2)$ & $\mathrm{C} 2-\mathrm{C} 3$ & $1.3560(14)$ \\
\hline $\mathrm{O} 3-\mathrm{C} 4$ & $1.2168(13)$ & $\mathrm{C} 4-\mathrm{C} 5$ & $1.4612(14)$ \\
\hline $\mathrm{O} 4-\mathrm{C} 6$ & $1.3264(12)$ & $\mathrm{C} 4-\mathrm{C} 6^{\mathrm{ii}}$ & $1.5045(15)$ \\
\hline $\mathrm{O} 4-\mathrm{H} 4$ & $0.86(2)$ & $\mathrm{C} 5-\mathrm{C} 6$ & $1.3504(15)$ \\
\hline $\mathrm{O} 5-\mathrm{C} 7$ & $1.2609(13)$ & $\mathrm{C} 8-\mathrm{C} 9$ & $1.5348(15)$ \\
\hline $\mathrm{N} 1-\mathrm{C} 7$ & $1.3338(14)$ & $\mathrm{C} 8-\mathrm{H} 8 \mathrm{~A}$ & 0.9900 \\
\hline $\mathrm{N} 1-\mathrm{C} 8$ & $1.4455(15)$ & $\mathrm{C} 8-\mathrm{H} 8 \mathrm{~B}$ & 0.9900 \\
\hline $\mathrm{N} 1-\mathrm{H} 1 \mathrm{~N}$ & $0.85(2)$ & $\mathrm{C} 9-\mathrm{H} 9 \mathrm{~A}$ & 0.9900 \\
\hline $\mathrm{N} 2-\mathrm{C} 7$ & $1.3397(14)$ & C9- $\mathrm{H} 9 \mathrm{~B}$ & 0.9900 \\
\hline $\mathrm{C} 3-\mathrm{O} 2-\mathrm{H} 2$ & $114.1(14)$ & $\mathrm{C} 6-\mathrm{C} 5-\mathrm{Cl} 2$ & $121.94(8)$ \\
\hline $\mathrm{C} 6-\mathrm{O} 4-\mathrm{H} 4$ & $115.8(13)$ & $\mathrm{C} 4-\mathrm{C} 5-\mathrm{Cl} 2$ & $117.13(8)$ \\
\hline $\mathrm{C} 7-\mathrm{N} 1-\mathrm{C} 8$ & $112.92(10)$ & $\mathrm{O} 4-\mathrm{C} 6-\mathrm{C} 5$ & $122.18(10)$ \\
\hline $\mathrm{C} 7-\mathrm{N} 1-\mathrm{H} 1 \mathrm{~N}$ & $121.2(15)$ & $\mathrm{O} 4-\mathrm{C} 6-\mathrm{C}^{\mathrm{ii}}$ & $117.46(9)$ \\
\hline $\mathrm{C} 8-\mathrm{N} 1-\mathrm{H} 1 \mathrm{~N}$ & $125.7(15)$ & $\mathrm{C} 5-\mathrm{C} 6-\mathrm{C} 4^{\mathrm{ii}}$ & $120.35(9)$ \\
\hline $\mathrm{C} 7-\mathrm{N} 2-\mathrm{C} 9$ & $112.46(10)$ & $\mathrm{O} 5-\mathrm{C} 7-\mathrm{N} 1$ & $125.87(10)$ \\
\hline $\mathrm{C} 7-\mathrm{N} 2-\mathrm{H} 2 \mathrm{~N}$ & $119.3(12)$ & $\mathrm{O} 5-\mathrm{C} 7-\mathrm{N} 2$ & $125.24(10)$ \\
\hline $\mathrm{C} 9-\mathrm{N} 2-\mathrm{H} 2 \mathrm{~N}$ & $127.5(12)$ & $\mathrm{N} 1-\mathrm{C} 7-\mathrm{N} 2$ & $108.88(10)$ \\
\hline $\mathrm{O} 1-\mathrm{C} 1-\mathrm{C} 2$ & $123.17(9)$ & $\mathrm{N} 1-\mathrm{C} 8-\mathrm{C} 9$ & $102.65(9)$ \\
\hline $\mathrm{O} 1-\mathrm{C} 1-\mathrm{C} 3^{\mathrm{i}}$ & $117.64(9)$ & $\mathrm{N} 1-\mathrm{C} 8-\mathrm{H} 8 \mathrm{~A}$ & 111.2 \\
\hline $\mathrm{C} 2-\mathrm{C} 1-\mathrm{C} 3^{\mathrm{i}}$ & $119.19(8)$ & $\mathrm{C} 9-\mathrm{C} 8-\mathrm{H} 8 \mathrm{~A}$ & 111.2 \\
\hline $\mathrm{C} 3-\mathrm{C} 2-\mathrm{C} 1$ & $121.28(9)$ & $\mathrm{N} 1-\mathrm{C} 8-\mathrm{H} 8 \mathrm{~B}$ & 111.2 \\
\hline $\mathrm{C} 3-\mathrm{C} 2-\mathrm{C} 11$ & $121.68(8)$ & $\mathrm{C} 9-\mathrm{C} 8-\mathrm{H} 8 \mathrm{~B}$ & 111.2 \\
\hline $\mathrm{C} 1-\mathrm{C} 2-\mathrm{C} 11$ & $117.03(7)$ & $\mathrm{H} 8 \mathrm{~A}-\mathrm{C} 8-\mathrm{H} 8 \mathrm{~B}$ & 109.2 \\
\hline $\mathrm{O} 2-\mathrm{C} 3-\mathrm{C} 2$ & $122.48(9)$ & $\mathrm{N} 2-\mathrm{C} 9-\mathrm{C} 8$ & $102.92(9)$ \\
\hline $\mathrm{O} 2-\mathrm{C} 3-\mathrm{C}^{\mathrm{i}}$ & $117.99(9)$ & $\mathrm{N} 2-\mathrm{C} 9-\mathrm{H} 9 \mathrm{~A}$ & 111.2 \\
\hline $\mathrm{C} 2-\mathrm{C} 3-\mathrm{C}^{\mathrm{i}}$ & $119.53(9)$ & $\mathrm{C} 8-\mathrm{C} 9-\mathrm{H} 9 \mathrm{~A}$ & 111.2 \\
\hline $\mathrm{O} 3-\mathrm{C} 4-\mathrm{C} 5$ & $123.19(10)$ & $\mathrm{N} 2-\mathrm{C} 9-\mathrm{H} 9 \mathrm{~B}$ & 111.2 \\
\hline $\mathrm{O} 3-\mathrm{C} 4-\mathrm{C} 6^{\mathrm{ii}}$ & $118.08(10)$ & $\mathrm{C} 8-\mathrm{C} 9-\mathrm{H} 9 \mathrm{~B}$ & 111.2 \\
\hline
\end{tabular}




$\begin{array}{llll}\mathrm{C} 5-\mathrm{C} 4-\mathrm{C} 6 & \\ \mathrm{C} 6-\mathrm{C} 5-\mathrm{C} 4 & 118.73(9) & \mathrm{H} 9 \mathrm{~A}-\mathrm{C} 9-\mathrm{H} 9 \mathrm{~B} & 109.1 \\ \mathrm{O} 1-\mathrm{C} 1-\mathrm{C} 2-\mathrm{C} 3 & 120.92(9) & & \\ \mathrm{C} 3-\mathrm{C} 1-\mathrm{C} 2-\mathrm{C} 3 & -179.04(11) & \mathrm{C} 4-\mathrm{C} 5-\mathrm{C} 6-\mathrm{O} 4 & 179.73(10) \\ \mathrm{O} 1-\mathrm{C} 1-\mathrm{C} 2-\mathrm{C} 11 & 0.34(16) & \mathrm{C} 2-\mathrm{C} 5-\mathrm{C} 6-\mathrm{O} 4 & -1.31(16) \\ \mathrm{C} 3-\mathrm{C} 1-\mathrm{C} 2-\mathrm{C} 11 & -0.19(14) & \mathrm{C} 4-\mathrm{C} 5-\mathrm{C} 6-\mathrm{C} 4{ }^{\mathrm{ii}} & 0.61(17) \\ \mathrm{C} 1-\mathrm{C} 2-\mathrm{C} 3-\mathrm{O} 2 & 179.19(7) & \mathrm{C} 2-\mathrm{C} 5-\mathrm{C} 6-\mathrm{C} 4^{\mathrm{ii}} & 179.57(8) \\ \mathrm{C} 11-\mathrm{C} 2-\mathrm{C} 3-\mathrm{O} 2 & 179.32(9) & \mathrm{C} 8-\mathrm{N} 1-\mathrm{C} 7-\mathrm{O} 5 & -176.92(10) \\ \mathrm{C} 1-\mathrm{C} 2-\mathrm{C} 3-\mathrm{C} 1^{\mathrm{i}} & 0.52(15) & \mathrm{C} 8-\mathrm{N} 1-\mathrm{C} 7-\mathrm{N} 2 & 3.23(15) \\ \mathrm{C} 11-\mathrm{C} 2-\mathrm{C} 3-\mathrm{C} 1^{\mathrm{i}} & -0.34(16) & \mathrm{C} 9-\mathrm{N} 2-\mathrm{C} 7-\mathrm{O} 5 & -4.43(15) \\ \mathrm{O} 3-\mathrm{C} 4-\mathrm{C} 5-\mathrm{C} 6 & -179.14(7) & \mathrm{C} 9-\mathrm{N} 2-\mathrm{C} 7-\mathrm{N} 1 & -0.84(14) \\ \mathrm{C} 66^{\mathrm{ii}}-\mathrm{C} 4-\mathrm{C} 5-\mathrm{C} 6 & 178.94(11) & \mathrm{C} 7-\mathrm{N} 1-\mathrm{C} 8-\mathrm{C} 9 & 3.73(14) \\ \mathrm{O} 3-\mathrm{C} 4-\mathrm{C} 5-\mathrm{C} 2 & -0.60(17) & \mathrm{C} 7-\mathrm{N} 2-\mathrm{C} 9-\mathrm{C} 8 & -1.64(13) \\ \mathrm{C} 6{ }^{\mathrm{ii}}-\mathrm{C} 4-\mathrm{C} 5-\mathrm{C} 2 & -0.07(15) & \mathrm{N} 1-\mathrm{C} 8-\mathrm{C} 9-\mathrm{N} 2 & \\ \end{array}$

Symmetry codes: (i) $-x+1,-y,-z+1$; (ii) $-x,-y+1,-z+1$.

Hydrogen-bond geometry $\left(\AA,{ }^{\circ}\right)$

\begin{tabular}{lllll}
\hline$D-\mathrm{H} \cdots A$ & $D-\mathrm{H}$ & $\mathrm{H} \cdots A$ & $D \cdots A$ & $D-\mathrm{H} \cdots A$ \\
\hline $\mathrm{O} 2-\mathrm{H} 2 \cdots \mathrm{O} 5$ & $0.81(2)$ & $1.82(2)$ & $2.6090(11)$ & $164(2)$ \\
$\mathrm{O} 4-\mathrm{H} 4 \cdots \mathrm{O} 5$ & $0.86(2)$ & $1.88(2)$ & $2.6635(12)$ & $151.0(19)$ \\
$\mathrm{N} 1-\mathrm{H} 1 N^{\cdots}{ }^{\mathrm{iii}}$ & $0.85(2)$ & $2.06(2)$ & $2.9003(15)$ & $168(2)$ \\
$\mathrm{N} 2-\mathrm{H} 2 N \cdots \mathrm{O} 3{ }^{\text {ii }}$ & $0.85(2)$ & $2.06(2)$ & $2.8654(15)$ & $158.6(17)$ \\
\hline
\end{tabular}

Symmetry codes: (ii) $-x,-y+1,-z+1$; (iii) $-x+2,-y,-z+1$.

2,5-Dichloro-3,6-dihydroxy-1,4-benzoquinone-imidazolidine-2,4-dione (1/2) (II)

\section{Crystal data}

$\mathrm{C}_{6} \mathrm{H}_{2} \mathrm{Cl}_{2} \mathrm{O}_{4} \cdot 2 \mathrm{C}_{3} \mathrm{H}_{4} \mathrm{~N}_{2} \mathrm{O}_{2}$

$M_{r}=409.14$

Monoclinic, $C 2 / \mathrm{c}$

$a=19.5690(8) \AA$

$b=5.18661(10) \AA$

$c=16.6103(3) \AA$

$\beta=117.965(3)^{\circ}$

$V=1489.03(8) \AA^{3}$

$Z=4$

\section{Data collection}

Rigaku R-AXIS RAPIDII diffractometer

Detector resolution: 10.000 pixels $\mathrm{mm}^{-1}$

$\omega$ scans

Absorption correction: numerical

(NUMABS; Higashi, 1999)

$T_{\min }=0.808, T_{\max }=0.888$

14622 measured reflections
$F(000)=832.00$

$D_{\mathrm{x}}=1.825 \mathrm{Mg} \mathrm{m}^{-3}$

Mo $K \alpha$ radiation, $\lambda=0.71075 \AA$

Cell parameters from 13670 reflections

$\theta=3.3-30.2^{\circ}$

$\mu=0.49 \mathrm{~mm}^{-1}$

$T=180 \mathrm{~K}$

Block, brown

$0.49 \times 0.33 \times 0.24 \mathrm{~mm}$

2181 independent reflections

2029 reflections with $I>2 \sigma(I)$

$R_{\text {int }}=0.072$

$\theta_{\text {max }}=30.0^{\circ}, \theta_{\min }=4.1^{\circ}$

$h=-27 \rightarrow 27$

$k=-7 \rightarrow 7$

$l=-22 \rightarrow 23$ 


\section{Refinement}

Refinement on $F^{2}$

$R\left[F^{2}>2 \sigma\left(F^{2}\right)\right]=0.036$

$w R\left(F^{2}\right)=0.100$

$S=1.08$

2181 reflections

130 parameters

0 restraints

Primary atom site location: structure-invariant direct methods
Secondary atom site location: difference Fourier map

Hydrogen site location: mixed

$\mathrm{H}$ atoms treated by a mixture of independent and constrained refinement

$w=1 /\left[\sigma^{2}\left(F_{\mathrm{o}}^{2}\right)+(0.0633 P)^{2}+0.4572 P\right]$ where $P=\left(F_{\mathrm{o}}^{2}+2 F_{\mathrm{c}}^{2}\right) / 3$

$(\Delta / \sigma)_{\max }<0.001$

$\Delta \rho_{\max }=0.44$ e $\AA^{-3}$

$\Delta \rho_{\min }=-0.40$ e $\AA^{-3}$

\section{Special details}

Geometry. All esds (except the esd in the dihedral angle between two 1.s. planes) are estimated using the full covariance matrix. The cell esds are taken into account individually in the estimation of esds in distances, angles and torsion angles; correlations between esds in cell parameters are only used when they are defined by crystal symmetry. An approximate (isotropic) treatment of cell esds is used for estimating esds involving l.s. planes.

Refinement. Refinement was performed using all reflections. The weighted R-factor (wR) and goodness of fit (S) are based on $\mathrm{F}^{2}$. R-factor (gt) are based on $\mathrm{F}$. The threshold expression of $\mathrm{F}^{2}>2.0 \operatorname{sigma}\left(\mathrm{F}^{2}\right)$ is used only for calculating Rfactor $(\mathrm{gt})$.

Fractional atomic coordinates and isotropic or equivalent isotropic displacement parameters $\left(\AA^{2}\right)$

\begin{tabular}{lllll}
\hline & $x$ & $y$ & $z$ & $U_{\text {iso }} / U_{\text {eq }}$ \\
\hline C11 & $0.09683(2)$ & $1.02553(6)$ & $0.33978(2)$ & $0.02980(12)$ \\
O1 & $0.11596(5)$ & $1.47273(17)$ & $0.45960(6)$ & $0.0313(2)$ \\
O2 & $0.25729(5)$ & $0.82514(16)$ & $0.40485(6)$ & $0.02794(19)$ \\
O3 & $0.39430(5)$ & $0.57413(17)$ & $0.43836(6)$ & $0.02824(19)$ \\
O4 & $0.31705(4)$ & $-0.10296(18)$ & $0.23989(6)$ & $0.0296(2)$ \\
N1 & $0.46359(5)$ & $0.26895(19)$ & $0.40528(6)$ & $0.0269(2)$ \\
N2 & $0.33612(5)$ & $0.25929(18)$ & $0.32811(6)$ & $0.02336(19)$ \\
C1 & $0.17655(6)$ & $1.3650(2)$ & $0.47556(7)$ & $0.0229(2)$ \\
C2 & $0.18138(6)$ & $1.1410(2)$ & $0.42588(7)$ & $0.0228(2)$ \\
C3 & $0.25018(6)$ & $1.0302(2)$ & $0.44779(7)$ & $0.0223(2)$ \\
C4 & $0.39913(6)$ & $0.3869(2)$ & $0.39566(7)$ & $0.0226(2)$ \\
C5 & $0.35871(6)$ & $0.0519(2)$ & $0.29613(7)$ & $0.0229(2)$ \\
C6 & $0.44610(6)$ & $0.0494(2)$ & $0.34539(8)$ & $0.0255(2)$ \\
H6A & 0.466307 & -0.112237 & 0.380266 & $0.031^{*}$ \\
H6B & 0.467588 & 0.070964 & 0.302420 & $0.031^{*}$ \\
H1N & $0.5127(12)$ & $0.308(4)$ & $0.4484(15)$ & $0.054(5)^{*}$ \\
H2 & $0.3043(12)$ & $0.772(4)$ & $0.4269(14)$ & $0.049(5)^{*}$ \\
H2N & $0.2861(11)$ & $0.313(4)$ & $0.3055(13)$ & $0.045(5)^{*}$ \\
& & & & \\
\hline
\end{tabular}

Atomic displacement parameters $\left(\AA^{2}\right)$

\begin{tabular}{lllllll}
\hline & $U^{11}$ & $U^{22}$ & $U^{33}$ & $U^{12}$ & $U^{13}$ & $U^{23}$ \\
\hline C11 & $0.02000(16)$ & $0.03480(18)$ & $0.02881(17)$ & $-0.00423(8)$ & $0.00662(12)$ & $-0.00241(9)$ \\
O1 & $0.0206(4)$ & $0.0327(4)$ & $0.0358(4)$ & $0.0052(3)$ & $0.0091(3)$ & $-0.0012(3)$ \\
O2 & $0.0237(4)$ & $0.0269(4)$ & $0.0314(4)$ & $0.0015(3)$ & $0.0114(3)$ & $-0.0036(3)$ \\
O3 & $0.0219(4)$ & $0.0300(4)$ & $0.0288(4)$ & $0.0015(3)$ & $0.0085(3)$ & $-0.0058(3)$
\end{tabular}




$\begin{array}{lllllll}\mathrm{O} 4 & 0.0198(4) & 0.0324(4) & 0.0321(4) & -0.0038(3) & 0.0085(3) & -0.0086(3) \\ \mathrm{N} 1 & 0.0160(4) & 0.0312(5) & 0.0288(4) & -0.0018(3) & 0.0067(3) & -0.0071(4) \\ \mathrm{N} 2 & 0.0158(4) & 0.0268(4) & 0.0239(4) & 0.0003(3) & 0.0063(3) & -0.0025(3) \\ \mathrm{C} 1 & 0.0191(4) & 0.0254(5) & 0.0226(4) & 0.0009(3) & 0.0084(4) & 0.0032(4) \\ \text { C2 } & 0.0177(4) & 0.0257(5) & 0.0220(4) & -0.0009(3) & 0.0069(3) & 0.0020(4) \\ \text { C3 } & 0.0205(4) & 0.0232(5) & 0.0226(4) & 0.0002(3) & 0.0094(4) & 0.0021(3) \\ \text { C4 } & 0.0180(4) & 0.0256(5) & 0.0221(4) & -0.0010(3) & 0.0076(3) & 0.0003(4) \\ \text { C5 } & 0.0173(4) & 0.0262(5) & 0.0239(5) & -0.0002(3) & 0.0086(4) & -0.0002(4) \\ \text { C6 } & 0.0162(4) & 0.0276(5) & 0.0295(5) & -0.0008(3) & 0.0079(4) & -0.0049(4)\end{array}$

Geometric parameters $\left(\stackrel{A}{\circ}{ }^{\circ}\right)$

\begin{tabular}{|c|c|c|c|}
\hline $\mathrm{C} 11-\mathrm{C} 2$ & $1.7094(10)$ & $\mathrm{N} 2-\mathrm{C} 5$ & $1.3620(14)$ \\
\hline $\mathrm{O} 1-\mathrm{C} 1$ & $1.2222(12)$ & $\mathrm{N} 2-\mathrm{C} 4$ & $1.3857(13)$ \\
\hline $\mathrm{O} 2-\mathrm{C} 3$ & $1.3237(13)$ & $\mathrm{N} 2-\mathrm{H} 2 \mathrm{~N}$ & $0.912(18)$ \\
\hline $\mathrm{O} 2-\mathrm{H} 2$ & $0.86(2)$ & $\mathrm{C} 1-\mathrm{C} 2$ & $1.4536(15)$ \\
\hline $\mathrm{O} 3-\mathrm{C} 4$ & $1.2318(14)$ & $\mathrm{C} 1-\mathrm{C} 3^{\mathrm{i}}$ & $1.5035(14)$ \\
\hline $\mathrm{O} 4-\mathrm{C} 5$ & $1.2117(13)$ & $\mathrm{C} 2-\mathrm{C} 3$ & $1.3475(14)$ \\
\hline $\mathrm{N} 1-\mathrm{C} 4$ & $1.3425(13)$ & $\mathrm{C} 5-\mathrm{C} 6$ & $1.5106(14)$ \\
\hline $\mathrm{N} 1-\mathrm{C} 6$ & $1.4436(14)$ & C6-H6A & 0.9900 \\
\hline $\mathrm{N} 1-\mathrm{H} 1 \mathrm{~N}$ & $0.91(2)$ & $\mathrm{C} 6-\mathrm{H} 6 \mathrm{~B}$ & 0.9900 \\
\hline $\mathrm{C} 3-\mathrm{O} 2-\mathrm{H} 2$ & $112.8(14)$ & $\mathrm{O} 2-\mathrm{C} 3-\mathrm{Cl}^{\mathrm{i}}$ & $116.58(9)$ \\
\hline $\mathrm{C} 4-\mathrm{N} 1-\mathrm{C} 6$ & $111.82(8)$ & $\mathrm{C} 2-\mathrm{C} 3-\mathrm{C} 1^{\mathrm{i}}$ & $120.65(9)$ \\
\hline $\mathrm{C} 4-\mathrm{N} 1-\mathrm{H} 1 \mathrm{~N}$ & $125.3(13)$ & $\mathrm{O} 3-\mathrm{C} 4-\mathrm{N} 1$ & $127.75(10)$ \\
\hline $\mathrm{C} 6-\mathrm{N} 1-\mathrm{H} 1 \mathrm{~N}$ & $122.5(13)$ & $\mathrm{O} 3-\mathrm{C} 4-\mathrm{N} 2$ & $124.29(9)$ \\
\hline $\mathrm{C} 5-\mathrm{N} 2-\mathrm{C} 4$ & $111.40(8)$ & $\mathrm{N} 1-\mathrm{C} 4-\mathrm{N} 2$ & $107.95(9)$ \\
\hline $\mathrm{C} 5-\mathrm{N} 2-\mathrm{H} 2 \mathrm{~N}$ & $124.3(12)$ & $\mathrm{O} 4-\mathrm{C} 5-\mathrm{N} 2$ & $126.87(10)$ \\
\hline $\mathrm{C} 4-\mathrm{N} 2-\mathrm{H} 2 \mathrm{~N}$ & $124.2(12)$ & $\mathrm{O} 4-\mathrm{C} 5-\mathrm{C} 6$ & $126.41(10)$ \\
\hline $\mathrm{O} 1-\mathrm{C} 1-\mathrm{C} 2$ & $123.86(10)$ & $\mathrm{N} 2-\mathrm{C} 5-\mathrm{C} 6$ & $106.71(9)$ \\
\hline $\mathrm{O} 1-\mathrm{C} 1-\mathrm{C}^{\mathrm{i}}$ & $117.46(10)$ & $\mathrm{N} 1-\mathrm{C} 6-\mathrm{C} 5$ & $102.06(8)$ \\
\hline $\mathrm{C} 2-\mathrm{C} 1-\mathrm{C}^{\mathrm{i}}$ & $118.67(8)$ & $\mathrm{N} 1-\mathrm{C} 6-\mathrm{H} 6 \mathrm{~A}$ & 111.4 \\
\hline $\mathrm{C} 3-\mathrm{C} 2-\mathrm{C} 1$ & $120.68(9)$ & $\mathrm{C} 5-\mathrm{C} 6-\mathrm{H} 6 \mathrm{~A}$ & 111.4 \\
\hline $\mathrm{C} 3-\mathrm{C} 2-\mathrm{Cl} 1$ & $121.94(8)$ & $\mathrm{N} 1-\mathrm{C} 6-\mathrm{H} 6 \mathrm{~B}$ & 111.4 \\
\hline $\mathrm{C} 1-\mathrm{C} 2-\mathrm{Cl1}$ & $117.37(7)$ & $\mathrm{C} 5-\mathrm{C} 6-\mathrm{H} 6 \mathrm{~B}$ & 111.4 \\
\hline $\mathrm{O} 2-\mathrm{C} 3-\mathrm{C} 2$ & $122.77(10)$ & $\mathrm{H} 6 \mathrm{~A}-\mathrm{C} 6-\mathrm{H} 6 \mathrm{~B}$ & 109.2 \\
\hline $\mathrm{O} 1-\mathrm{C} 1-\mathrm{C} 2-\mathrm{C} 3$ & $-179.33(10)$ & $\mathrm{C} 6-\mathrm{N} 1-\mathrm{C} 4-\mathrm{N} 2$ & $-1.64(12)$ \\
\hline $\mathrm{C} 33^{\mathrm{i}}-\mathrm{C} 1-\mathrm{C} 2-\mathrm{C} 3$ & $-0.32(16)$ & $\mathrm{C} 5-\mathrm{N} 2-\mathrm{C} 4-\mathrm{O} 3$ & $-176.84(11)$ \\
\hline $\mathrm{O} 1-\mathrm{C} 1-\mathrm{C} 2-\mathrm{C} 11$ & $0.48(15)$ & $\mathrm{C} 5-\mathrm{N} 2-\mathrm{C} 4-\mathrm{N} 1$ & $2.61(12)$ \\
\hline $\mathrm{C} 33^{\mathrm{i}}-\mathrm{C} 1-\mathrm{C} 2-\mathrm{C} 11$ & $179.49(7)$ & $\mathrm{C} 4-\mathrm{N} 2-\mathrm{C} 5-\mathrm{O} 4$ & $176.75(11)$ \\
\hline $\mathrm{C} 1-\mathrm{C} 2-\mathrm{C} 3-\mathrm{O} 2$ & $179.56(9)$ & $\mathrm{C} 4-\mathrm{N} 2-\mathrm{C} 5-\mathrm{C} 6$ & $-2.45(12)$ \\
\hline $\mathrm{C} 11-\mathrm{C} 2-\mathrm{C} 3-\mathrm{O} 2$ & $-0.24(15)$ & $\mathrm{C} 4-\mathrm{N} 1-\mathrm{C} 6-\mathrm{C} 5$ & $0.19(12)$ \\
\hline $\mathrm{C} 1-\mathrm{C} 2-\mathrm{C} 3-\mathrm{C}^{\mathrm{i}}$ & $0.32(16)$ & $\mathrm{O} 4-\mathrm{C} 5-\mathrm{C} 6-\mathrm{N} 1$ & $-177.85(11)$ \\
\hline $\mathrm{C} 11-\mathrm{C} 2-\mathrm{C} 3-\mathrm{C}^{\mathrm{i}}$ & $-179.48(7)$ & $\mathrm{N} 2-\mathrm{C} 5-\mathrm{C} 6-\mathrm{N} 1$ & $1.35(11)$ \\
\hline $\mathrm{C} 6-\mathrm{N} 1-\mathrm{C} 4-\mathrm{O} 3$ & $177.78(11)$ & & \\
\hline
\end{tabular}

Symmetry code: (i) $-x+1 / 2,-y+5 / 2,-z+1$. 
Hydrogen-bond geometry $\left(A,{ }^{\circ}\right)$

\begin{tabular}{lllll}
\hline$D-\mathrm{H} \cdots A$ & $D-\mathrm{H}$ & $\mathrm{H} \cdots A$ & $D \cdots A$ & $D-\mathrm{H} \cdots A$ \\
\hline $\mathrm{O} 2-\mathrm{H} 2 \cdots \mathrm{O} 3$ & $0.86(2)$ & $1.97(2)$ & $2.7917(15)$ & $160(2)$ \\
$\mathrm{N} 1-\mathrm{H} 1 N \cdots \mathrm{O} 3^{\mathrm{ii}}$ & $0.91(2)$ & $2.00(2)$ & $2.8927(13)$ & $165(2)$ \\
$\mathrm{N} 2-\mathrm{H} 2 N \cdots \mathrm{O} 44^{\text {iii }}$ & $0.91(2)$ & $1.85(2)$ & $2.7560(14)$ & $176(2)$
\end{tabular}

Symmetry codes: (ii) $-x+1,-y+1,-z+1$; (iii) $-x+1 / 2, y+1 / 2,-z+1 / 2$. 